\begin{tabular}{||ll||}
\hline Citation/Reference & $\begin{array}{l}\text { Andersen K.T., Moonen M., "Adaptive time-frequency analysis for noise } \\
\text { reduction in an audio filter bank with low delay", IEEE Transactions on } \\
\text { audio, speech and language processing, vol. 24, no. 4, Apr. 2016, pp. 784- } \\
795 .\end{array}$ \\
\hline Archived version & Final publisher's version / pdf \\
\hline Published version & $\underline{\text { http://dx.doi.org/10.1109/TASLP.2016.252779 }}$ \\
\hline Journal homepage & $\underline{\text { http://ieexplore.iee.org/xpl/RecentIssue.jsp?punumber=10376. }}$ \\
\hline IR & $+\underline{\text { https://lirias.kuleuven.be/handle/123456789/527103 }}$ \\
\hline
\end{tabular}

(article begins on next page) 


\title{
Adaptive Time-Frequency Analysis for Noise Reduction in an Audio Filter Bank With Low Delay
}

\author{
Kristian Timm Andersen, Student Member, IEEE, and Marc Moonen, Fellow, IEEE
}

\begin{abstract}
In this paper, an adaptive time-frequency analysis scheme is proposed along with a synthesis scheme using an asymmetric window. The proposed scheme is suitable for audio noise reduction with a low delay in the range of 0 to $4 \mathrm{~ms}$. The main novelty of the paper is the adaptive analysis scheme that can adapt to the incoming signal independently in both time and frequency by employing a complex filter on a DFT modulated filter bank. A number of adaptive time-frequency schemes are described that are suitable for low delay and low computational complexity. The adaptive time-frequency scheme is used for the computation of noise reduction gain factors, which are then adopted in a nonadaptive analysis/synthesis scheme. The synthesis scheme uses an asymmetric window to achieve a good tradeoff between low delay and a sharp frequency response. Examples are given of the adaptive analysis and measurements of the synthesis scheme are given to show that the filter bank has a gain dependent nonlinear phase response. Finally, a noise reduction task is performed that shows good performance compared to reference implementations in terms of segmental SNR and PESQ.
\end{abstract}

Index Terms-Adaptive time-frequency analysis, noise reduction, speech enhancement, low delay.

\section{INTRODUCTION}

$\mathbf{O}$ $\mathrm{NE}$ of the most important tasks for a real-time audio device is to present a clear and audible signal to the user at a low delay. For a hearing aid in particular, a low delay is critical since sound traveling through the vent into the ear canal should not get too out of sync with the sound coming from the hearing aid speaker. Studies have shown that delays exceeding approximately $10 \mathrm{~ms}$ can be objectionable while delays around 3-5 ms can still be detected [1]. This delay includes buffering and would also include A/D and D/A conversion. Similar results are also found in [2]. In this paper we consider low delay to mean a filter bank that can apply a frequency dependent gain to the signal in around $4 \mathrm{~ms}$ or less. This delay is not a hard limit, since the total delay also includes buffering and

Manuscript received August 10, 2015; revised November 27, 2015 and January 22, 2016; accepted January 23, 2016. Date of publication February 08, 2016; date of current version March 08, 2016. This work was supported by the Danish Agency for Science, Technology, and Innovation and conducted in collaboration between KU Leuven and Widex A/S through the industrial Ph.D. program (case number 13-135472). The associate editor coordinating the review of this manuscript and approving it for publication was Prof. Simon Doclo.

K. T. Andersen is with the Department of Electrical Engineering (ESATSTADIUS), KU Leuven, 3001 Leuven, Belgium, and also with Widex A/S, 3540 Lynge, Denmark (e-mail: kristian@esat.kuleuven.be).

M. Moonen is with the Department of Electrical Engineering (ESATSTADIUS), KU Leuven, 3001 Leuven, Belgium (e-mail: Marc.Moonen@ esat.kuleuven.ne)

Color versions of one or more of the figures in this paper are available online at http://ieeexplore.ieee.org.

Digital Object Identifier 10.1109/TASLP.2016.2526779 computational delay, although these delays should be small in comparison to ensure a truly low delay implementation.

The quality of the signal can be improved using noise reduction, which in the single-channel case is done by applying a frequency-dependent gain to the signal. To achieve some tradeoff between time and frequency resolution in the noise reduction, the filter bank is often designed to have a nonuniform frequency resolution with more narrow bands in the low frequencies. A popular framework for nonuniform frequency resolution is the wavelet transform [3], for instance the critically sampled tree-structured filter bank. For our application, however, the iterated use of the so-called "mother" wavelet results in a high group delay that makes it inappropriate for very low delay applications. Also, the need for noise reduction in the filter bank necessitates oversampling to avoid aliasing in the reconstruction [4], [5]. A frequency warped low delay filter bank that can approximate the Bark frequency scale has been proposed in [6] and [7].

A number of adaptive time-frequency (TF) resolution schemes have been suggested, see for instance [8], [9] and the references therein. Also, it has been shown that adapting the TF resolution can lead to improvements in noise reduction [10], [11]. Common for these approaches is that they are not suitable for low delay implementations since they require longer time windows to determine the TF resolution and/or that they have a high computational complexity. A window switching approach to adaptive TF resolution with a low delay of $10 \mathrm{~ms}$ has been proposed in [12]. An alternative approach where the TF resolution is smoothed over time has also been proposed [13], [14].

The approach in this paper differs from these methods in that an adaptive TF analysis that can adapt its resolution independently in both time and frequency is developed which is suitable for low delay implementation and has a low computational complexity. The adaptive TF analysis is incorporated into the framework of the DFT modulated filter bank and paired with a synthesis that is, again, suitable for low delay implementation. The adaptive TF analysis is used to calculate the gain factors, while the synthesis uses the underlying DFT filter bank. This keeps the delay of the filter bank itself at $4 \mathrm{~ms}$ or below, and all parameter estimation is done with this delay constraint.

The paper is structured as follows. In section II, the underlying basis function of the adaptive TF analysis is derived and it is shown that the adaptive TF analysis can be realized as a filtering on top of a DFT modulated filter bank. In section III, the estimation of the filter coefficients for the adaptive TF analysis is described. It is shown how the bandwidth of a frequency band can be calculated from the filter coefficients, which makes it possible to design a nonuniform analysis with any desired 
bandwidth for each individual frequency band. It is shown how the analysis filter bank can be time-varying and a number of different adaptive TF analysis schemes are described. An example is given to compare the different adaptive TF analysis schemes. In section IV, the synthesis using an asymmetric window is described. It is shown how the asymmetric window achieves a tradeoff between low delay and sufficient band attenuation. Further reduction in the delay is achieved by applying the frequency dependent gain for the noise reduction as a finite impulse response (FIR) filter, either by reusing the asymmetric window or by calculating the minimum phase response. The section ends with a discussion of the synthesis scheme with some experimental results. Section V contains the conclusion and future outlook.

\section{Adaptive Time-Frequency Analysis Scheme}

Assume an overcomplete set of $L$ basis functions:

$$
g_{k}[n]=h[n] W_{L}^{k n}, \quad 0 \leq k \leq L-1
$$

where $h[n]$ is a suitable real-valued window with a low-pass characteristic and with length $N(N<L)$ that localizes the basis functions in time and $W_{L}=e^{2 \pi j / L}$. A time-domain signal $x[n]$ is mapped to the transform domain by the convolution between $x[n]$ and $g_{k}[n]$ and decimated with a factor $R(R<$ $N)$ :

$$
\begin{aligned}
x_{k}[i]=\left(g_{k} * x\right)[i R] & =\sum_{n=-\infty}^{\infty} g_{k}[n] x[i R-n] \\
& =\sum_{n=0}^{L-1} h[L-n] x[i R+n-L] e^{-2 \pi j n k / L}
\end{aligned}
$$

where $*$ is the convolution operator. Thus, the transformation is calculated with the discrete Fourier transform (DFT), $X_{k, i}=$ $x_{k}[i]$ is the short-time Fourier transform (STFT) with hop-size $R, N$ is the frame length and $L$ is the number of frequency bands. Throughout this paper we will use the values $L=512$, $N=64, R=16$ for examples, but it is possible to use other values depending on the application.

Equation (2) can be interpreted as the signal $x[n]$ being filtered with frequency modulated versions of the low-pass filter $h[n]$, which has given rise to the term DFT modulated filter bank. This is efficiently implemented using the fast Fourier transform (FFT). A combined analysis/synthesis scheme representation is seen in Figure 1. The input signal $X(z)$ is first filtered by $L$ band-pass filters $H_{k}(z)$ and then downsampled by $R$ to give $X_{k}(z)$. Each subband signal $X_{k}(z)$ is then processed, which in a noise reduction application is where the gain is applied. The processed subband signals are then upsampled by $R$, filtered with a synthesis filter and finally summed. This can be written as:

$$
y[m]=\sum_{i=-\infty}^{\infty} f[m-i R] \frac{1}{L} \sum_{k=0}^{L-1} Y_{k, i} e^{2 \pi j k(m-i R) / L}
$$

where $f[m]$ is a synthesis window and $Y_{k, i}$ is the signal to be reconstructed after processing. The analysis filters and synthesis filters correspond to frequency shifted versions of the

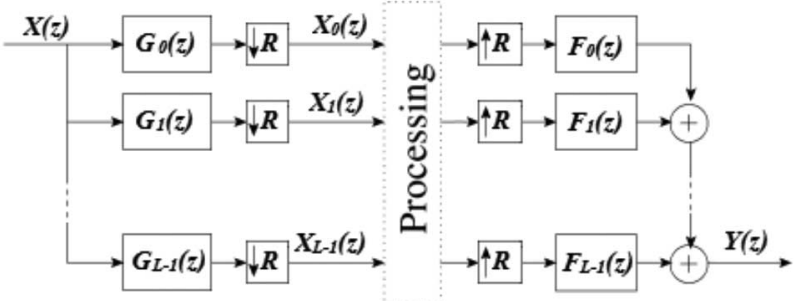

Fig. 1. Analysis/synthesis scheme.

low-pass analysis $h[n]$ and synthesis $f[n]$ windows respectively. A full treatment of DFT filter banks and multirate systems is outside the scope of this paper and we refer the interested reader to [5]. In this paper, since $N<L$, the transform domain representation has redundancy, which in a filter bank interpretation can be understood as a high degree of overlap between neighboring frequency bands.

A major limitation in a low-delay filter bank is the obtained frequency resolution resulting from the short analysis window $h[n]$. An improved low-pass characteristic can be obtained from $h[n]$ by filtering with a hop-size $R$ :

$$
\tilde{h}_{k, i}[n]=\sum_{q=0}^{Q} b_{k, i, q} h[n-q R]+\sum_{p=1}^{P} a_{k, i, p} \tilde{h}_{k, i-p}[n-p R]
$$

where $b_{k, i, q}$ and $a_{k, i, p}$ are filter coefficients that are variable in both frequency band $k$ and time $i . \tilde{h}_{k, i}[n]$ is a longer window than $h[n]$ and therefore has a more narrow low-pass frequency response. The improved basis functions are then:

$$
\tilde{g}_{k, i}[n]=\tilde{h}_{k, i}[n] W_{L}^{n k}, \quad 0 \leq k \leq L-1
$$

which can offer improved frequency resolution, based on a window $\tilde{h}_{k, i}[n]$ that is less localized in time. Depending on the filter coefficients $a_{k, i, p}$ and $b_{k, i, q}$, the improved basis functions $\tilde{g}_{k, i}[n]$ can have infinite length and can be chosen independently for each frequency band $k$. Since a low delay and a low computational complexity is required, $Q$ and $P$ should be set to small values. In the following sections, we use $Q=0$ and $P=1$. $\tilde{g}_{k, i}[n]$ is sampled with the same time- and frequency steps as $g_{k}[n]$ and has essentially traded some redundancy in frequency for some redundancy in time. This redundant filtering scheme is what allows the analysis to adapt its resolution independently for each frequency band while maintaining a low delay.

The improved STFT is given by:

$$
\begin{aligned}
\tilde{x}_{k}[i]= & \sum_{n=-\infty}^{\infty} \tilde{g}_{k, i}[n] x[i R-n] \\
= & \sum_{q=0}^{Q} b_{k, i, q} \sum_{n=-\infty}^{\infty} h[n-q R] x[i R-n] W_{L}^{n k} \\
& +\sum_{p=1}^{P} a_{k, i, p} \sum_{n=-\infty}^{\infty} \tilde{h}_{k, i-p}[n-p R] x[i R-n] W_{L}^{n k} \\
= & \sum_{q=0}^{Q} b_{k, i, q} x_{k}[i-q] W_{L}^{q R k}+\sum_{p=1}^{P} a_{k, i, p} \tilde{x}_{k}[i-p] W_{L}^{p R k}
\end{aligned}
$$




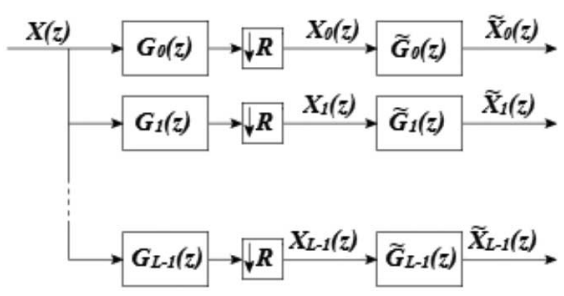

Fig. 2. Adaptive TF analysis scheme.

It is seen that $\tilde{x}_{k}[i]$ can be obtained as a filtering of $x_{k}[i]$ without the explicit calculation of the underlying basis functions. However, (4) and (5) are useful for properly setting the coefficients of the adaptive TF analysis, as explained in the next section. The low computational complexity of the adaptive TF analysis is achieved by using (6) to calculate $\tilde{x}_{k}[i]$. The proposed adaptive TF analysis scheme can be seen in Figure 2. In this paper, the adaptive TF analysis is used for the computation of noise reduction gain factors, which are then adopted in a non-adaptive (hence fixed delay) analysis-synthesis scheme, see also Figure 6.

\section{Setting the Filter Coefficients for Adaptive TIME-FREQUENCY ANALYSIS}

The analysis presented in section II allows for the design of an individual analysis window in each frequency band using any filter design method as long as all the windows share the same underlying basis function $h[n]$. In this section we consider how to set the filter coefficients for the adaptive TF analysis. In section III-A we motivate the use of a simple first-order auto-regressive filter for the adaptive TF analysis. In section III-B we consider the estimation of the filter coefficients for a time-invariant filter and in section III-C we consider a number of adaptive TF analysis schemes for a time-varying filter. Section III-D contains some experimental results obtained using the discussed adaptive TF analysis schemes.

\section{A. Setting the Filter Coefficients Using a First-Order Auto- Regressive Filter}

As we are interested in a low delay, low complexity analysis, we consider the estimation of the filter coefficients using only one auto-regressive coefficient, i.e. $P=1, Q=0$. This is the lowest order filter that can be used to improve the frequency resolution, while the auto-regressive coefficient gives the possibility to set the effective length of $\tilde{h}_{k, i}[n]$ exemplified by the exponential decay of a first-order auto-regressive filter. This choice makes it possible to derive simple settings of the filter coefficients and leaves two coefficients to be estimated for each frequency band. To maintain the DC gain of $\tilde{h}_{k, i}[n]$ over time, the two coefficients are set to $b_{k, i, 0}=\alpha_{k, i}, a_{k, i, 1}=1-\alpha_{k, i}$ :

$$
\tilde{h}_{k, i}[n]=\alpha_{k, i} h[n]+\left(1-\alpha_{k, i}\right) \tilde{h}_{k, i-1}[n-R]
$$

where $0<\alpha_{k, i} \leq 1$.

To see that this maintains the DC gain of $\tilde{h}_{k, i}[n]$, we evaluate the discrete-time Fourier transform (DTFT) of $\tilde{h}_{k, i}[n]$ at $\omega=0$ :

$$
\tilde{H}_{k, i}(0)=\alpha_{k, i} H(0)+\left(1-\alpha_{k, i}\right) \tilde{H}_{k, i-1}(0) e^{-j R 0}
$$

where

$$
\tilde{H}_{k, i}(\omega)=\sum_{n=-\infty}^{\infty} \tilde{h}_{k, i}[n] e^{-j \omega n}
$$

is the DTFT of $\tilde{h}_{k, i}[n]$.

Subtracting $H(0)$ from (8) and taking the absolute value gives:

$$
\left|\tilde{H}_{k, i}(0)-H(0)\right|=\left|1-\alpha_{k, i}\right|\left|\tilde{H}_{k, i-1}(0)-H(0)\right|
$$

Since $0<\alpha_{k, i} \leq 1$, it follows that either $\tilde{H}_{k, i}(0)=H(0)$ or $\left|\tilde{H}_{k, i}(0)-H(0)\right|$ decreases for every new $i$ and therefore that $\tilde{H}_{k, i}(0)=H(0)$ for $i \rightarrow \infty$. Since $\tilde{g}_{k, i}[n]$ is a frequency shifted version of $\tilde{h}_{k, i}[n]$, where $H(0)$ is shifted to the center frequency of the $k$ th frequency band, $\alpha_{k, i}$ does not change the energy at the center frequency of each frequency band compared to $g_{k}[n]$. Therefore the underlying basis functions can be interpreted as a time-varying analysis filter bank, where each frequency band has constant energy at its center frequency and $\alpha_{k, i}$ controls the bandwidth of frequency band $k$ at time $i$. Note that, in the timevarying case, the auto-regressive nature of the filter means that the bandwidth also depends on previous values of $\alpha_{k, i}$.

\section{B. Setting the Filter Coefficients for a Time-Invariant Filter}

In this section we consider the calculation of the filter coefficients $\alpha_{k}$ for a time-invariant filter of the form $b_{k, i, 0}=$ $\alpha_{k}, a_{k, i, 1}=1-\alpha_{k}$, i.e. where the filter is time-invariant (constant) for every frequency band. To define a measure of bandwidth we use the distance between the $3 \mathrm{~dB}$ cutoff points, that is we specify a desired bandwidth $\omega_{k}=\frac{\pi B_{k}}{f_{s}}$ for each frequency band, where $B_{k}$ is the bandwidth and $f_{s}$ is the sampling rate in $\mathrm{Hz}$.

Evaluating the squared magnitude of the DTFT of the filter $\tilde{H}_{k}\left(\omega_{k}\right)$ using (4) for the specific setting of $a_{k, i, 1}$ and $b_{k, i, 0}$, we can write:

$$
\left|\tilde{H}_{k}\left(\omega_{k}\right)\right|^{2}=\frac{\alpha_{k}^{2}\left|H\left(\omega_{k}\right)\right|^{2}}{1-2\left(1-\alpha_{k}\right) \cos <\left(\omega_{k} R\right)+\left(1-\alpha_{k}\right)^{2}}
$$

To find $\alpha_{k}$ for a given bandwidth $\omega_{k}$, we set $\left|\tilde{H}_{k}\left(\omega_{k}\right)\right|^{2}=$ $\frac{|H(0)|^{2}}{2}$ and rewrite the equation as a quadratic function of $\alpha_{k}$ :

$$
\alpha_{k}^{2} c_{1}+\alpha_{k} c_{2}-c_{2}=0
$$

where

$$
\begin{aligned}
& c_{1}=\frac{\left|H\left(\omega_{k}\right)\right|^{2}}{|H(0)|^{2}}-\frac{1}{2} \\
& c_{2}=1-\cos <\left(\omega_{k} R\right)
\end{aligned}
$$

The solution is:

$$
\alpha_{k}=\frac{c_{2}}{c_{1}}\left(\sqrt{\frac{c_{1}}{c_{2}}+\frac{1}{4}}-\frac{1}{2}\right)
$$

where the solution for negative $\alpha_{k}$ has been discarded, as $\alpha_{k}$ must be positive. $\frac{\left|H\left(\omega_{k}\right)\right|^{2}}{|H(0)|^{2}}$ is calculated from the DTFT of the 

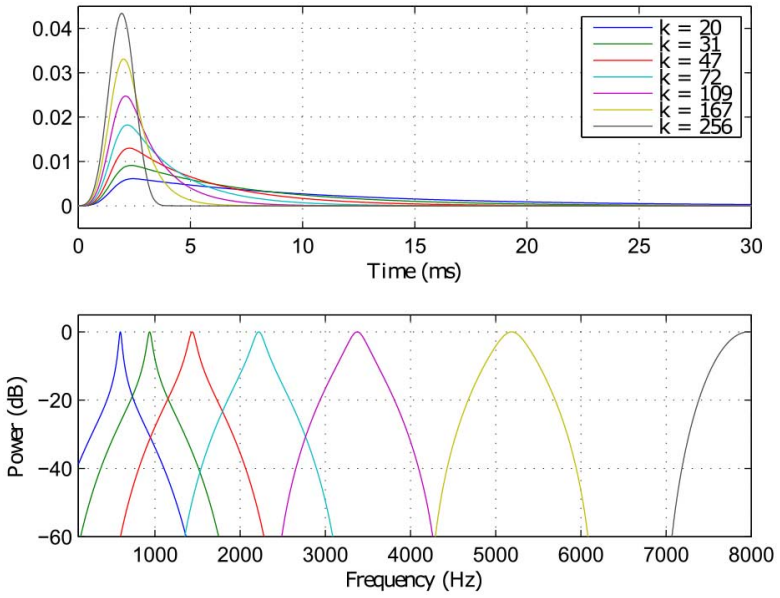

Fig. 3. 7 basis functions for a constant Q-transform with a Blackmann-Harris window $\left(N=64, L=512, R=16, f_{s}=16 \mathrm{kHz}\right)$ ). (Top) Improved analysis windows $\tilde{h}_{k}[n]$. (Bottom) Power spectrum of improved basis functions $\tilde{G}_{k}(\omega)$.

used window $h[n]$ and $\omega_{k}$ should be chosen small enough so that $\frac{\left|H\left(\omega_{k}\right)\right|^{2}}{|H(0)|^{2}}>\frac{1}{2}$. This is clear, as the new $3 \mathrm{~dB}$ cutoff point must be smaller than that of $h[n]$.

Equation (15) can be used to design a filter bank with a desired bandwidth for each frequency band. As an example, a constant-Q filter bank can be designed by setting $B_{k}$ to be proportional to $k$ :

$$
B_{k}=\max \left(\frac{2 k B_{H}}{L}, \frac{f_{s}}{2 L}\right)
$$

where $B_{H}$ is the $3 \mathrm{~dB}$ bandwidth for $H(\omega) . B_{k}$ is bounded downwards by $\frac{f_{s}}{2 L}$ to ensure that the cutoff point of a frequency band is not smaller than half the distance to the neighbor band. An example of the basis functions for $h[n]$ equal to a BlackmanHarris window is seen in Figure 3. The frequency bands are linearly spaced on the frequency axis, but to avoid clutter in the plot only 7 logarithmically spaced basis functions are shown. It is seen that $\tilde{h}_{k}[n]$ is an asymmetric window with most of the energy concentrated at the most recent part of the signal and an exponential decay that depends on the parameter $\alpha_{k}$. This asymmetry means that the analysis is mostly determined by the most recent part of the signal and makes the analysis more appropriate for low delay processing compared to symmetric basis functions that arise from for instance wavelets. It is seen that a smaller value of $\alpha_{k}$ in the lower frequencies sharpens the main lobe of $\tilde{G}_{k}(\omega)$. Further sharpening could be achieved by using a higher order filter, but then at the expense of the peak of $\tilde{h}_{k}[n]$ moving further backwards in time. The proposed TF analysis scheme is also significantly cheaper than processing the full filter in the time domain and the use of a single coefficient for each frequency band means that each frequency band only requires one complex and one real multiplication per time update to obtain a nonuniform TF resolution.

\section{Setting the Filter Coefficients for a Time-Varying Filter}

In this section we consider the calculation of filter coefficients for a time-varying filter. More specifically, we develop three simple estimators for a time-varying $\mathrm{TF}$ analysis that are suitable for low delay and low complexity implementation. Many different adaptive TF analysis schemes have been suggested, but, to the best of our knowledge, no scheme exists that is suitable for both low delay and low complexity implementation and at the same time allows for full adaptability in both time and frequency.

Following the use of a first-order auto-regressive filter as in section III-A, the adaptive TF analysis can also be used for a time-varying filter:

$$
\tilde{X}_{k, i}=\alpha_{k, i} X_{k, i}+\left(1-\alpha_{k, i}\right) \tilde{X}_{k, i-1} W_{L}^{R k}
$$

Under the assumption that the signal can be decomposed into quasi-periodic signal components that can be separated in the combined TF domain, a simple optimal estimator can be derived.

In previous sections, it was shown that the energy of $\tilde{H}_{k, i}(0)$ is constant and equal to $\mathrm{H}(0)$ for $i \rightarrow \infty$ and any value of $\alpha_{k, i}$. Consequently, for a sinusoid with frequency $\frac{2 \pi k}{L}$, the energy of $\tilde{X}_{k, i}$ is invariant to the choice of $\alpha_{k, i}$. Therefore any increase in energy, due to the change of $\alpha_{k, i}$, must come from signal components not centered on $\tilde{X}_{k, i}$. Consequently, an optimal estimate of $\alpha_{k, i}$ is the value $\hat{\alpha}_{k, i}$ :

$$
\hat{\alpha}_{k, i}=\underset{\alpha_{k, i}}{\arg \min }\left[\left|\tilde{X}_{k, i}\right|^{2}\right]
$$

subject to:

$$
\alpha_{\min } \leq \alpha_{k, i} \leq \alpha_{\max }
$$

where $\alpha_{\min }$ and $\alpha_{\max }$ are parameters that limits the TF resolution. A regularized version of this estimator is:

$$
\hat{\alpha}_{k, i}=\underset{\alpha_{k, i}}{\arg \min }\left[\left|\tilde{X}_{k, i}\right|^{2}+\lambda_{k} \alpha_{k, i}\right]
$$

where $\lambda_{k}\left(\lambda_{k}>0\right)$ is a regularization parameter. The term $\lambda_{k} \alpha_{k, i}$ punishes large values of $\alpha_{k, i}$, which favors longer analysis windows for each basis function. This is justified since it improves the frequency resolution in the absence of a strongly defined minimum. Since $\left|\tilde{X}_{k, i}\right|^{2}$ is a convex quadratic function of $\alpha_{k, i}$, the minimum can be found by setting the derivative to zero:

$$
\frac{\partial\left[\left|\tilde{X}_{k, i}\right|^{2}+\lambda_{k} \alpha_{k, i}\right]}{\partial \alpha_{k, i}}=0
$$

which gives:

$$
\begin{aligned}
\hat{\alpha}_{k, i} & =\min \left(\max \left(\bar{\alpha}_{k, i}, \alpha_{\min }\right), \alpha_{\max }\right) \\
\bar{\alpha}_{k, i} & =-\frac{\operatorname{Re}\left\{\tilde{X}_{k, i-1} W_{L}^{R k}\left(X_{k, i}-\tilde{X}_{k, i-1} W_{L}^{R k}\right)^{*}\right\}+\lambda_{k}}{\left|X_{k, i}-\tilde{X}_{k, i-1} W_{L}^{R k}\right|^{2}}
\end{aligned}
$$

where $\operatorname{Re}\{x\}$ is the real part of $x$. This estimator gives the (regularized) TF analysis that minimizes the energy in each frequency band. Since the underlying basis function has constant energy for $\tilde{H}_{k, i}(0)$, this estimator minimizes the energy leaking into $X_{k, i}$. 

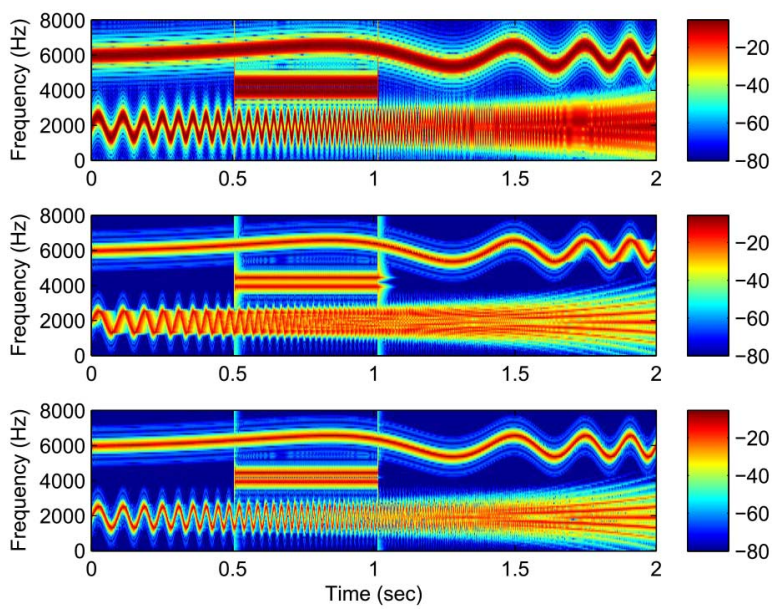

Fig. 4. Frequency modulated sinusoids. (Top) Short analysis window $h[n]$, $\alpha_{k}=1$. (Middle) Long analysis window, $\alpha_{k}=0.1$. (Bottom) Improved adaptive analysis window.

An example of this estimator is seen in Figure 4. The signal consists of a number of sinusoids that have been frequency modulated to a varying degree. The top plot shows the analysis with $\alpha_{k}=1$, which corresponds to the analysis with only the short window $h[n]$. The middle plot shows the analysis with $\alpha_{k}=0.1$ and the bottom plot shows the analysis using the proposed estimator $\left(\lambda_{k}=1 e^{-5}, \alpha_{\min }=0.1, \alpha_{\max }=1\right)$. It is seen that the top plot has poor frequency resolution due to the short window, while having a good time resolution. The middle plot has a good frequency resolution and can discriminate between two closely spaced sinusoids while having a poor time resolution, which results in smearing of the sinusoids over time. It is also seen in the right part of the plot that when the modulations become so fast that they have several periods within the length of the window, the modulation is seen as a periodic function itself and is represented as harmonics of the underlying carrier. This illustrates the ambiguity that the same signal can be seen as fundamentally different depending on the window length. In this case, we prefer the longer window, since it is a more sparse representation, i.e. it represents the signal using fewer signal components. In the lower plot it is seen that the adaptive TF analysis gives a superior time- and frequency-resolution compared to the two fixed-resolution analyses and can adapt to each sinusoid independently without causing smearing over time. Unlike most adaptive TF analysis schemes, which can only adapt in either time or frequency, the proposed method can adapt to several signal components individually as long as they are separated in the combined TF domain.

An alternative estimator is given in [15] and [16] where it is shown that a minimax cross-entropy estimate of the squared magnitude TF distribution can be found as the minimum value of a set of $M$ squared magnitude TF distributions at each $(k, i)$ coordinate :

$$
\left|\hat{X}_{k, i}\right|^{2}=E \min _{m} S_{k, i, m}
$$

where $E$ is a normalization constant and $S_{k, i, m}$ is the set of $M$ squared magnitude TF distributions $\left\{S_{k, i, m}: m \in\right.$
$(1, \ldots, M), M \geq 2\}$ calculated with equal-energy basis functions. In our case, the $M$ different squared magnitude $\mathrm{TF}$ distributions are given by:

$$
S_{k, i, m}=\frac{\left|\tilde{X}_{k, i, m}\right|^{2}}{E_{\tilde{h}_{k, i, m}}}
$$

where

$$
\tilde{X}_{k, i, m}=\alpha_{m} X_{k, i}+\left(1-\alpha_{m}\right) \tilde{X}_{k, i-1, m} W_{L}^{R k}
$$

is the TF distribution for $\alpha_{m}$ and $E_{\tilde{h}_{k, i, m}}$ is the energy of the underlying window function that can be precomputed from (4) with $b_{k, i, 0}=\alpha_{m}, a_{k, i, 1}=1-\alpha_{m}$ and other coefficients set to zero. Thus, the minimax cross-entropy estimator is obtained by calculating $M \mathrm{TF}$ distributions and taking the minimum of the set of squared magnitude equal-energy windowed TF distributions in each $(k, i)$ coordinate. This is in contrast to the estimator in (22) where there is no normalization of the energy before estimation. Since the energy normalization $E$ depends on the value $\alpha_{m}$, finding the global minimum of the energy, as in (18) and (20), is a non-convex problem and therefore computationally infeasible to solve in real-time. This is avoided by resorting to a minimax cross-entropy estimate instead of the true optimal solution to the minimization problem. It should also be clear that choosing a TF distribution from a limited set of precomputed TF distributions does not give a smooth TF analysis as in Figure 4.

The preceding estimators assume that the signal can be decomposed into signal components that are non-overlapping in the TF domain. For signals that are contaminated by high levels of noise, this is not a reasonable assumption and in this case we revert to a simpler estimator that uses a non-stationarity test to decide what resolution should be used. If a non-stationarity is detected, the TF distribution is calculated from the short window $h[n]$ :

$$
\tilde{X}_{k, i}=\alpha_{k} X_{k, i}
$$

Otherwise, the TF distribution is updated using equation (17). Following a non-stationarity, the support of the underlying basis function $\tilde{g}_{k}[n]$ grows after each update, which results in a narrower bandwidth in the frequency domain. After a while it converges to the stationary basis function derived in section III-B depending on what value of $\alpha_{k}$ is used. It is noted that before the basis function has converged to the stationary case, the energy of the basis function will grow with time. However, if the value of $\alpha_{k}$ is known in advance, the energy and shape of the basis function can be precomputed and stored in a look-up table.

The non-stationarity test is based on the criteria from [17] which we adapted to our purpose in [18]:

$$
\begin{aligned}
\mathrm{LR} & =\sqrt{R} e^{-\frac{1}{2}(R-1)} \\
R & =\frac{\left|X_{k, i}\right|^{2} /\left(\alpha_{k}^{2} E_{h}\right)}{\left|\tilde{X}_{k, i-1}\right|^{2} / E_{\tilde{h}_{k, i-1}}}
\end{aligned}
$$

where $E_{h}$ is the energy of $h[n]$ and $E_{\tilde{h}_{k, i-1}}$ is the energy of the underlying window function at time $i-1$. LR is compared to a 

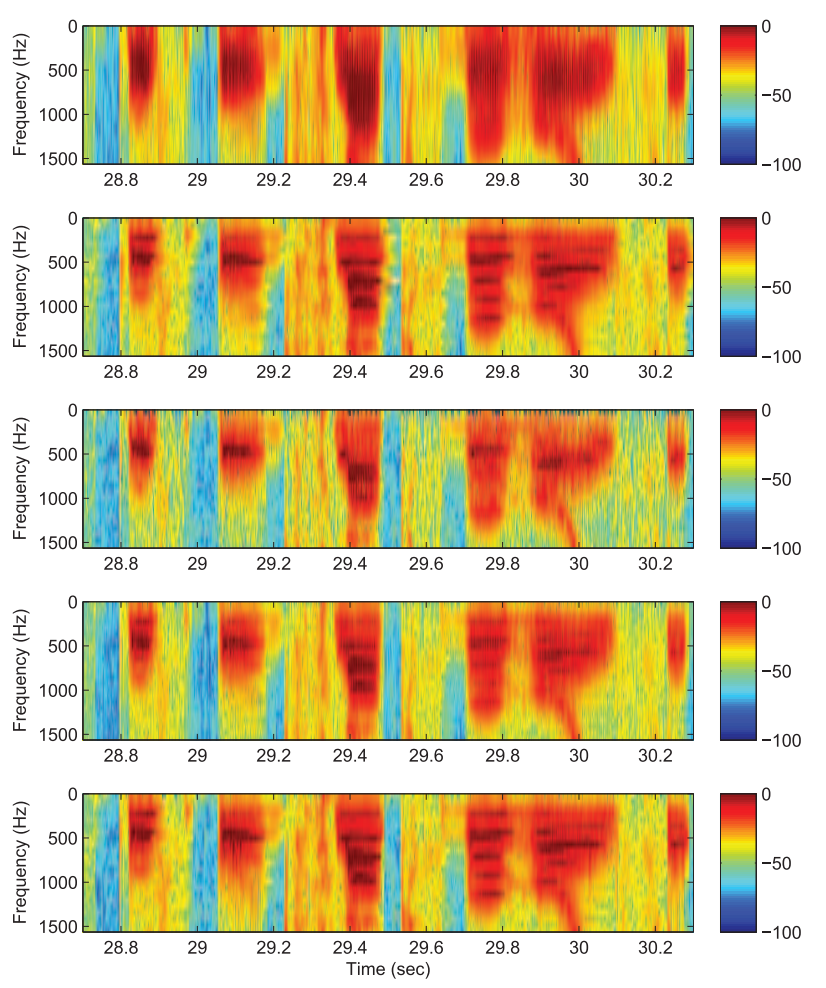

Fig. 5. Speech signal processed with 5 different TF analysis schemes. In descending order, (1) TF analysis using the short window $h[n]$, (2) Constant $\mathrm{Q}$ transform TF analysis, (3) Adaptive TF analysis that minimizes the energy, (4) Adaptive minimax cross-entropy TF analysis and (5) Adaptive TF analysis using a non-stationarity criteria.

threshold value $\lambda$ and if $\mathrm{LR}<\lambda$ a non-stationarity is detected. To improve the robustness of the estimate, a non-stationarity is only detected if $\mathrm{V}$ adjacent frequency bands fail the test.

\section{Discussion on Adaptive Time-Frequency Analysis}

In section III-B and III-C different TF analysis schemes have been described. A time-invariant analysis filter scheme that allows a specified bandwidth to be set for each frequency band as well as three adaptive schemes have been presented. A requirement for all the presented schemes is that they must be computationally simple and have a low delay to allow for a real-time implementation in an audio processing device such as a hearing aid. The low computational complexity is achieved by employing a first order filter on top of an oversampled DFT modulated filter bank and the low delay follows from the short analysis window $h[n]$ of length $N$ and the exponential decay of the filter response that puts emphasis on the most recent part of the signal.

To compare the described TF distributions, a speech signal was processed through each of the different TF analysis schemes. The results are seen in Figure 5. The figure shows the low frequency area of the speech signal where the harmonics can be resolved by the TF analysis schemes, which illustrates the improved frequency resolution. The constant $\mathrm{Q}$ analysis scheme, however, has a poor time resolution due to the long analysis window. Especially it is seen that the speech energy is smeared in time at the end of the speech components due to the exponential decay of the analysis windows illustrating the well-known trade-off between time and frequency resolution. The three adaptive TF analysis schemes all have an improved frequency resolution compared to the short window $h[n]$ while also having a much better time resolution than the constant $\mathrm{Q}$ analysis scheme. This is because they detect the end of the speech signal and change to the short analysis window. The computational complexity of the analysis is shown in Table I. The DFT is considered to be implemented using a real FFT algorithm [19]. The added complexity of the proposed adaptive analysis is the cost of calculating the adaptive filter for the improved analysis and the complexity factor shows the relative number of multiplications and additions of the adaptive analysis compared to the DFT analysis filter bank for the values used in the examples. It is seen that, especially for large $L$, it is the FFT algorithm that dominates the computational complexity of the proposed method and an exact calculation of the cost therefore depends on what FFT algorithm is available on the chosen hardware platform.

\section{Synthesis With Low Delay}

The TF analysis presented in section II and III corresponds to an overcomplete transform of the signal, and therefore there is an infinite number of ways to reconstruct the signal from the analysis. There are numerous articles on perfect reconstruction modulated filter banks and other similar filter bank structures, see for instance [20], [21] and [5]. However, in many real-time audio processing applications it is not the reconstruction per se that is of interest, but the ability to apply a certain processing, for instance to apply a specified gain to each individual frequency band, and to do so with a low delay. This processing is time-varying and requires oversampling in the filter bank to avoid aliasing as in [22], [23] or [24]. The proposed adaptive $\mathrm{TF}$ analysis presented in this paper already has oversampling built into it. In this section, we focus on how to apply a desired gain to each individual frequency band with a low delay, by using an asymmetric synthesis window. The asymmetric window results in a non-linear phase response when a gain is applied to each individual frequency band, but since the ear is relatively insensitive to small phase distortions, there is no perceptual degradation of the signal. In the following, we disregard delays due to input/output buffering and processing of the data and measure the delay by the effective length of the combined analysis and synthesis window. Furthermore, we use the asymmetric window in an FIR filter design to further reduce the delay to N/2, similar to the filter bank equalizer in [7]. This is compared to a minimum phase FIR filter that represents the lowest possible delay for a given gain in each individual frequency band.

Since the adaptive TF analysis uses an individual analysis basis function in each frequency band, it would also require an individual synthesis basis function in each frequency band. However, since the adaptive TF analysis is calculated as a first order minimum-phase auto-regressive (AR) filtering of $h[n]$, this filtering itself is in theory invertible, although there could be numerical problems with trying to invert a time-varying AR filter. However, since the input to the AR filter is known apriori, it can be excluded from the signal path and then only 
TABLE I

Computational Complexity for the Proposed Analysis Scheme

\begin{tabular}{|c|c|c|c|}
\hline analysis scheme & real multiplications & real additions & complexity factor \\
\hline DFT analysis filter bank & $\frac{1}{R}\left(\frac{L}{2} \log _{2} L-\frac{3 L}{2}+2+N\right)$ & $\frac{1}{R}\left(\frac{3 L}{2} \log _{2} L-\frac{5}{2} L+4\right)$ & 1 \\
Adaptive analysis & $\frac{5 L}{2 R}$ & $\frac{8 L}{2 R}$ & $\sim 0.46$ \\
\hline
\end{tabular}

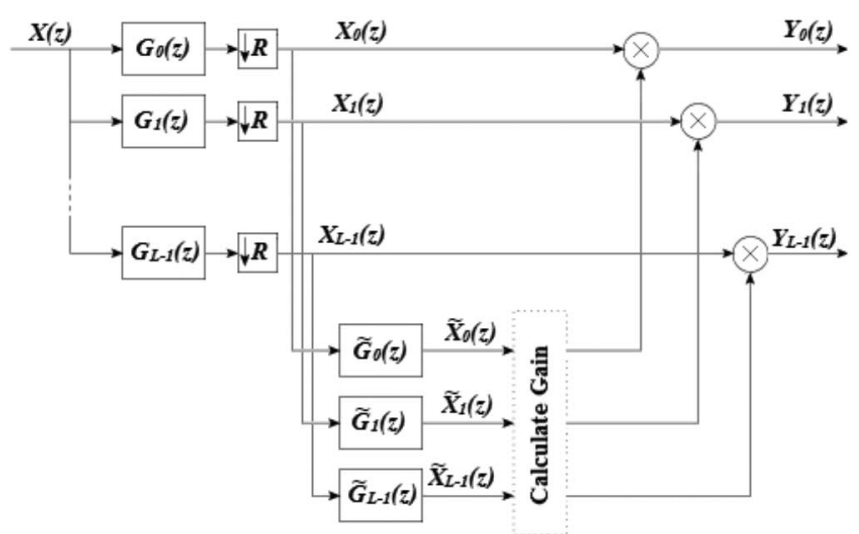

Fig. 6. Analysis scheme where the adaptive TF analysis is used to calculate a gain that is then applied to the primary signal path.

used to calculate a frequency dependent gain $G$ vector applied to $X_{k}(z)$ as seen in Figure 6. Removing the adaptive TF analysis from the signal path also greatly simplifies the design of the synthesis window and will be followed in the rest of this section.

\section{A. Synthesis Using an Assymmetric Window}

Following Figure 1 and inserting the DFT-modulated synthesis formula (2) into (3), without applying a gain i.e. $Y_{k, i}=$ $X_{k, i}$, the criterion for perfect reconstruction (PR) of the input signal is derived as:

$$
\sum_{i=-\infty}^{\infty} h[m-i R] f[m-i R]=1
$$

where it is used that $h[n]$ has length $N<L$. This condition is met for Hann windows for $R=2^{a}, a \in \mathbf{N}$ and under similar conditions for many other windows such as the higher-order generalized cosine windows. This requirement only ensures perfect reconstruction and does not imply that there is sufficient filtering being done by the windows. Several proposals have been made for optimizing these windows, while sometimes settling for near-perfect reconstruction, see for instance [25], [26] or [27].

An asymmetric window can be decomposed into the three following components:

$$
\boldsymbol{h}_{F}[n]=\left[\mathbf{0}^{T}, \boldsymbol{h}_{l h}[n]^{T}, \boldsymbol{h}_{r h}[n]^{T}\right]^{T}
$$

where $\mathbf{0}$ is a zero vector of size $(L-N) / 2, \boldsymbol{h}_{l h}[n]$ is the left half of a window and $\boldsymbol{h}_{r h}[n]$ is the right half of a window. The effective length of the window is the length of $\boldsymbol{h}_{l h}[n]$ and $\boldsymbol{h}_{r h}[n]$ which we denote with $W$. An asymmetric window of
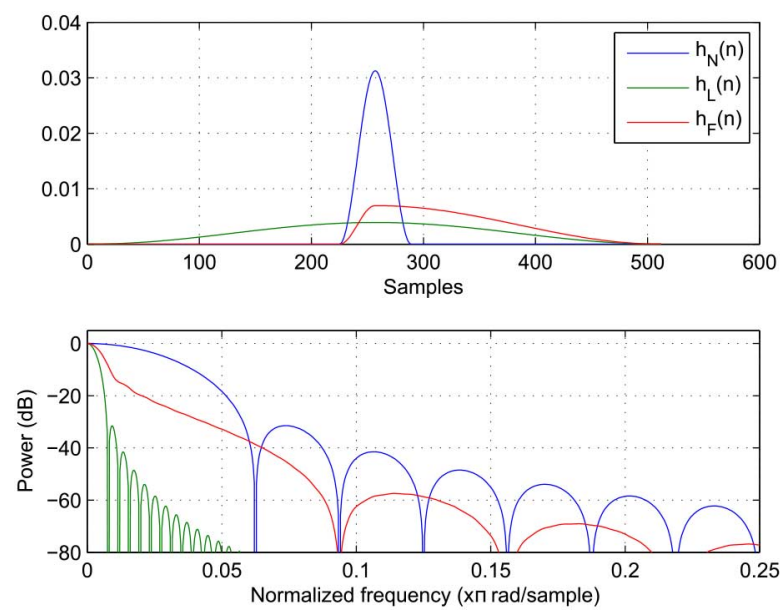

Fig. 7. Time domain (Top) and frequency domain (Bottom) representation of two symmetric Hann windows $h_{N}[n]$ and $h_{L}[n]$ and the asymmetric window $h_{F}[n]$

this type is seen in Figure 7 along with two symmetric Hann windows of length $N$ and $L$ respectively. The asymmetric window consists of the zero vector $\mathbf{0}$, the left half of a Hann window of length $N$ and the right half of a Hann window of length $L$.

Setting the synthesis window equal to the analysis window $f[n]=h[n]=h_{N}[n]$ would achieve a delay of $N$ samples and fulfills the PR criterion, but the resulting filtering would be poor. Setting $f[n]=h_{L}[n]$ achieves a higher degree of filtering in the synthesis stage as seen in the lower part of Figure 7. This comes at the price of an increased delay equal to the length of the combined analysis and synthesis window $(L+N) / 2$. Using the asymmetric window $f[n]=h_{F}[n]$ maintains the low delay of $N$ samples, equivalent to the delay of $h_{N}[n]$, with a better filtering. The PR criteria is only fulfilled approximately, however as seen in the following paragraph, the overall frequency response only exhibits minor ripples.

To measure the response of the three different synthesis windows, white noise is sent through the analysis/synthesis scheme and the output is measured. The response is estimated by deconvolving the output with the input using division in the frequency domain and is seen in Figure 8. It is seen that all three synthesis windows lead to a flat overall frequency response with less than $0.01 \mathrm{~dB}$ ripple and that $f[n]=h_{L}[n]$ indeed leads to the predicted delay of $(L+N) / 2=288$ samples while the two other synthesis windows leads to an equal delay of $N=64$. If we assume a sample rate of $16 \mathrm{kHz}$, this corresponds to a delay of $18 \mathrm{~ms}$ and $4 \mathrm{~ms}$ respectively. The response of one frequency band is seen in Figure 9. It is seen that $f[n]=h_{L}[n]$ and $f[n]=$ $h_{N}[n]$ leads to a symmetric temporal response which is due to the symmetric analysis and synthesis windows. The sharper frequency response with $f[n]=h_{L}[n]$ compared to $f[n]=h_{N}[n]$ 

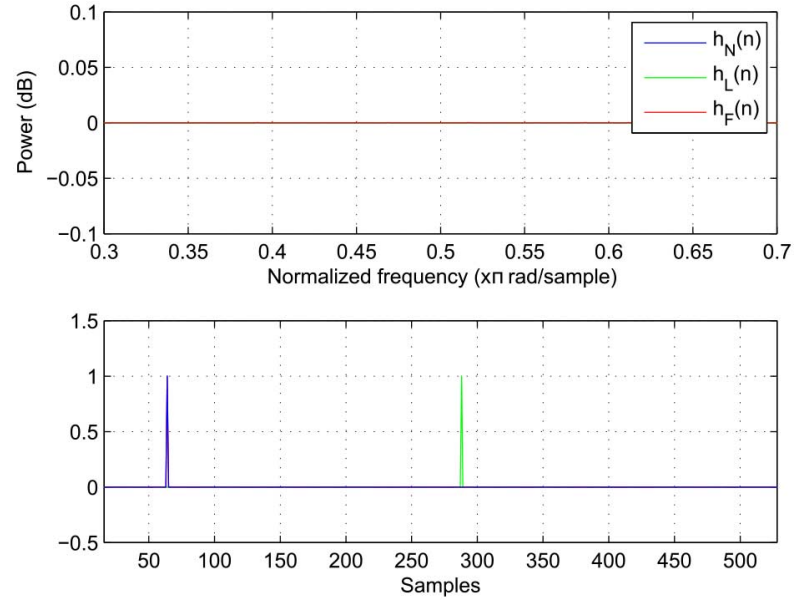

Fig. 8. Overall output of the analysis/synthesis scheme. (Top) Power spectrum. (Bottom) Temporal response. The blue impulse is on top of the red one.
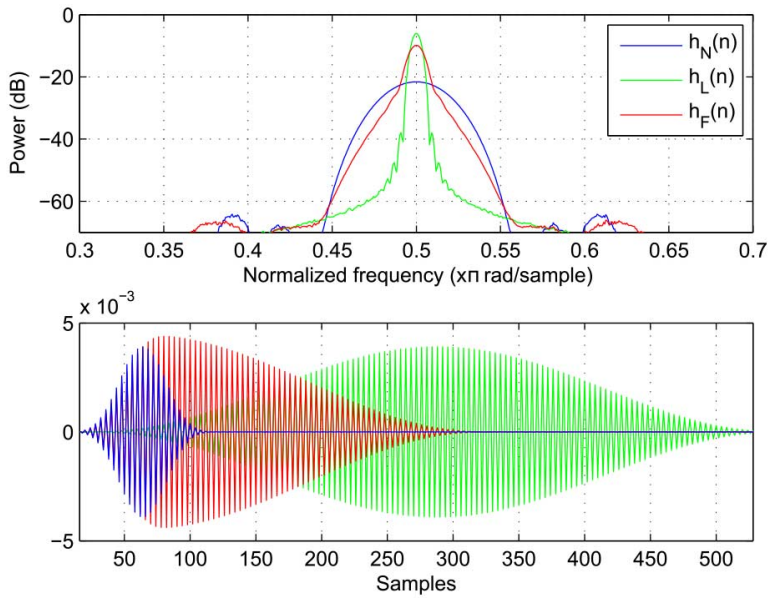

Fig. 9. Output of one frequency band in the analysis/synthesis scheme. (Top) Power spectrum. (Bottom) Temporal response.

comes at the price of the increased delay. $f[n]=h_{F}[n]$ leads to an asymmetric temporal response where the degree of asymmetry depends on how sharp the frequency response is. Thus, we characterize the analysis/synthesis system with synthesis windows $h_{L}[n]$ or $h_{N}[n]$ as linear phase filter banks and using synthesis window $h_{F}[n]$ as a mixed phase filter bank where the phase shift depends on the gain that is applied to the frequency bands (it is clearly not minimum phase due to the minimum delay of $N$ samples).

\section{B. Further Reduction in Delay Using FIR Filter and Minimum Phase Estimation}

The asymmetric synthesis window used in the previous section reduces the delay to $N$ samples by setting the first $(L-N) / 2$ samples of the synthesis window to zero. If a further reduction of the delay is needed, a parallel signal path without the R-fold decimation can be used, where the frequency dependent gain is applied to the signal as seen in Figure 10. The gain is applied to the signal by transforming it to the time-domain using a IDFT and filtering the signal with a corresponding FIR filter [28]. In this case, the gain can be interpreted

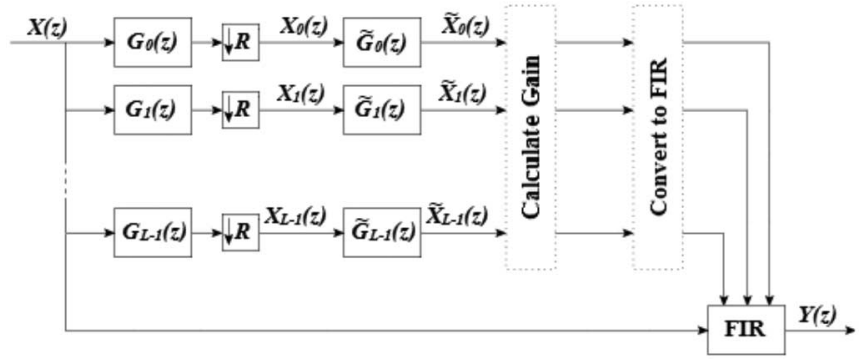

Fig. 10. Adaptive TF analysis where the gain is applied in a parallel signal path with a time-varying FIR filter.

as the magnitude response of the FIR filter. The delay for a timedomain filter, using a symmetric window of length $N$ is $N / 2$. Using an asymmetric window would in this case also provide a way to maintain the low delay of $N / 2$ while giving a better filtering than a symmetric window. However, an even further reduction of the delay can be achieved by calculating the minimum phase filter for a given magnitude response. A minimum phase filter has the property of minimum group delay, which means that the energy is maximally concentrated in the low delay coefficients. Infinite impulse response (IIR) filters calculated by solving the set of Yule-Walker equations have been proposed in the context of speech enhancement [29], but minimum phase can equally well be realized using FIR filters, which is usually simpler to deal with when using time-varying filters, since stability is not an issue.

The gain vector $\boldsymbol{G}$ of length $L$ is equal to the magnitude response of the desired minimum phase filter:

$$
\boldsymbol{H}_{\min }=\left|\boldsymbol{H}_{\min }\right| e^{j \Theta}=\boldsymbol{G} e^{j \Theta}
$$

where $\boldsymbol{H}_{\min }$ is the minimum phase frequency response vector and $\Theta$ is the phase vector that must be estimated. The filter coefficients are found by taking the IDFT of $\boldsymbol{H}_{\min }$ and then low-pass filtering each coefficient over time to smooth it. The phase $\Theta$ can be found by calculating the zeros of the corresponding linear phase response and then reflecting the zeros that are outside the unit circle inside the unit circle [30]. This method however is prone to numerical inaccuracies, especially for large filter orders. Instead, an approximate nonparametric method involving the cepstrum is used. It has been shown [31] that $\Theta$ can be calculated as:

$$
\Theta=-j D F T[\boldsymbol{s} \cdot I D F T[\log \boldsymbol{G}]]
$$

where

$$
s(k)= \begin{cases}0 & \text { if } k=0, k=L / 2, \\ 1 & \text { if } 0<k<L / 2, \\ -1 & \text { if } L / 2<k<L .\end{cases}
$$

The accuracy of this method depends on the length of the (I)DFT and how steep the cuts in the magnitude response are. In audio noise reduction, the gain is usually limited to a range between $\mathrm{Gmin}$ and $0 \mathrm{~dB}$, where $\mathrm{Gmin}$ is the minimum gain that is used. Setting Gmin to $-20 \mathrm{~dB}$ is usually sufficient to give good noise reduction and avoid noisy artifacts, and also prevents significant artifacts from the minimum phase estimation of $\Theta$. 
TABLE II

Comparison of Measured Delay for Different Methods

\begin{tabular}{|c|c|c|}
\hline method & window length $(W)$ & delay \\
\hline AAF & 288 & $4 \mathrm{~ms}$ \\
$\mathrm{AAN}$ & 64 & $4 \mathrm{~ms}$ \\
$\mathrm{AAL}$ & 512 & $18 \mathrm{~ms}$ \\
$\mathrm{FIR}_{A}$ & 288 & $2 \mathrm{~ms}$ \\
$\mathrm{FIR}_{\text {min }}$ & 128 & $0-1 \mathrm{~ms}$ \\
\hline
\end{tabular}

\section{Performance Analysis}

Table II shows a comparison of the synthesis schemes that have been discussed and lists the total delay for a $N=64$ analysis window at $16 \mathrm{kHz}$. The methods AAF, AAN and AAL corresponds to the adaptive analysis in Figure 10 followed by a synthesis using the windows $h_{F}(n), h_{N}(n)$ and $h_{L}(n)$ from Figure 7 respectively. The methods FIR $_{\mathrm{A}}$ and FIR $_{\min }$ corresponds to the adaptive analysis in Figure 10 where the FIR filter is using the asymmetric window and minimum phase filter described in section IV-B respectively. The window length $W$ is the length of the synthesis window and in the FIR filter case corresponds to the length of the FIR filter. The total delay of the analysis-synthesis scheme is found by calculating the crosscorrelation between the input and output in frames and finding the lag value with the highest correlation. The delay of $\mathrm{FIR}_{\min }$ is gain dependent, but has been found to be between 0 and 1 samples. Since FIR $_{\min }$ has most of the energy concentrated in the lowest order coefficients, its filter order is reduced to $W=2 N$ to save computations. This is found to have no significant impact on the output signal. The computational complexity of the different schemes can be seen in Table III. The DFT is considered to be computed with a real FFT algorithm [19] and the cost of calculating the FFT of a complex sequence of length $L$ is approximately double the cost of a real sequence of length $L$. All FFT's are applied to real sequences except the last inverse FFT to calculate the filter coefficients of the minimum phase filter FIR ${ }_{\min }$. The exact number of real multiplications and additions depends strongly on the used FFT algorithm, but the complexity factor shows the relative cost of the compared methods for the values used in the examples. The symbols $\mathcal{M}$ and $\mathcal{A}$ represent the multiplication and addition cost respectively of an exponential and a logarithm operation, whose values depend on the actual implementation. The coefficients of the FIR filters are smoothed over time using a first-order auto-regressive filter, which can be implemented using 1 multiplication and 2 additions per sample. It is seen that the lower delay of the FIR filters comes with the price of a significant added computational complexity due to processing the FIR filter at the full sample rate.

To evaluate the performance of each synthesis scheme for a noise reduction task, 100 speech sentences from the TIMIT database recorded at $16 \mathrm{kHz}$ were mixed with noise at different signal-to-noise ratios (SNR) and used as input to each of the described schemes. For the analysis, the following values were used: $L=512, N=64, R=16, h[n]$ is the Hann window and the adaptive TF analysis with non-stationarity detection was used with $\alpha_{k}=0.05$ and $\lambda=0.65$. The gain is calculated as the Wiener gain:

$$
G_{k, i}=\frac{\xi_{k, i}}{1+\xi_{k, i}}
$$

where $\xi_{k, i}$ is the a-priori SNR, which is calculated using the a-priori SNR smoother [32] with a smoothing parameter $a=$ 0.97. A minimum gain of $-15 \mathrm{~dB}$ is set to avoid significant noise distortion such as musical noise. The noise estimate is using an unbiased MMSE-based noise estimation method [33] that is suitable for real-time implementation. The gain coefficients are the same for all schemes, which only differ in the synthesis method. The average segmental SNR and PESQ value [34] is calculated and plotted in Figure 11 for a traffic noise signal. It should be noted that the use of SNR values for a noise reduction scheme that is not linear phase is not fully justified since it is not possible to align the input and output signals completely. This is clearly seen for FIR $_{\min }$ that performs significantly worse than any other scheme, which is indeed due to the minimum phase property of the filter. For FIR $\mathrm{min}_{\text {in }}$, the performance should be judged more on the perceptual quality which is measured by PESQ. It is seen that using AAL gives an improvement of about 1-2 dB in segmental SNR compared to AAN, at the cost of an added delay of $14 \mathrm{~ms}$. The asymmetric synthesis window in AAF gives a good tradeoff between the two symmetric windows with a low delay of $4 \mathrm{~ms}$ and about $0.5 \mathrm{~dB}$ SNR less than AAL. FIR $_{\mathrm{A}}$ has a very slight deterioration in performance compared to AAF. Looking at the PESQ values, AAL performs about $0.10-0.15$ better than AAN while AAF again has a good tradeoff between delay and performance. The FIR filters perform better in the perceptual test which is as expected. Informal listening tests have verified that, perceptually, AAN has more artifacts than the other output signals and creates more distortion of the speech.

The proposed AAF method has also been compared to a number of published methods with a similar low delay. The frequency warped FIR filter as described in [2], [6], (WFIR) gives a non-uniform frequency resolution approximating the Bark scale and according to the similar method in [7] achieves a similar performance to a Bark scaled analysis/synthesis filter bank. The frequency warping means the delay is frequency dependent and a phase-equalizer has been applied to the output to give a constant delay of $4 \mathrm{~ms}$, so a segmental SNR value can be calculated. We note that the results from [7] cannot be used directly in our comparison, since they use a lower sample rate of $8 \mathrm{kHz}$ and a delay of $8 \mathrm{~ms}$. A spectral subtraction method with reduced delay [13] (SSRD) is also used as comparison. Note that, although the gain convolution itself only has a delay of $2 \mathrm{~ms}$, the calculation of the gain function assumes a buffer delay of $20 \mathrm{~ms}$, so it is not low delay according to the definition used in this paper. This could be avoided by updating the $20 \mathrm{~ms}$ buffer and gain function every $2 \mathrm{~ms}$, which could affect the performance since the gain is then only dependent on previous data. An adaptive window-switching approach [17] (BSW), that uses a low- and high-frequency resolution analysis scheme, has been implemented with a comparable delay of $4 \mathrm{~ms}$. This is achieved by using a short analysis and synthesis window of only $4 \mathrm{~ms}$. The high frequency resolution window achieves a comparable frequency resolution to AAF. The high frequency resolution 
TABLE III

COMPARISON OF COMPUTATIONAL COMPLEXITY FOR DIFFERENT SYNTHESIS SCHEMES

\begin{tabular}{|c|c|c|c|}
\hline method & real multiplications & real additions & complexity factor \\
\hline DFT synthesis filter bank & $\frac{1}{R}\left(\frac{L}{2} \log _{2} L-\frac{L}{2}+2+W\right)$ & $\frac{1}{R}\left(\frac{3 L}{2} \log _{2} L-\frac{5}{2} L+4+W\right)$ & 1 \\
Asymmetric FIR filter & $\frac{1}{R}\left(\frac{L}{2} \log _{2} L-\frac{3 L}{2}+2+W\right)+2 W$ & $\frac{1}{R}\left(\frac{3 L}{2} \log _{2} L-\frac{5}{2} L+4\right)+3 W-1$ & $\sim 3.7$ \\
Minimum-phase FIR filter & $\frac{1}{R}\left(2 L \log _{2} L-6 L+8+\mathcal{M}\right)+2 W$ & $\frac{1}{R}\left(6 L \log _{2} L-10 L+16+\mathcal{A}\right)+3 W-1$ & $\sim 4.7$ \\
\hline
\end{tabular}
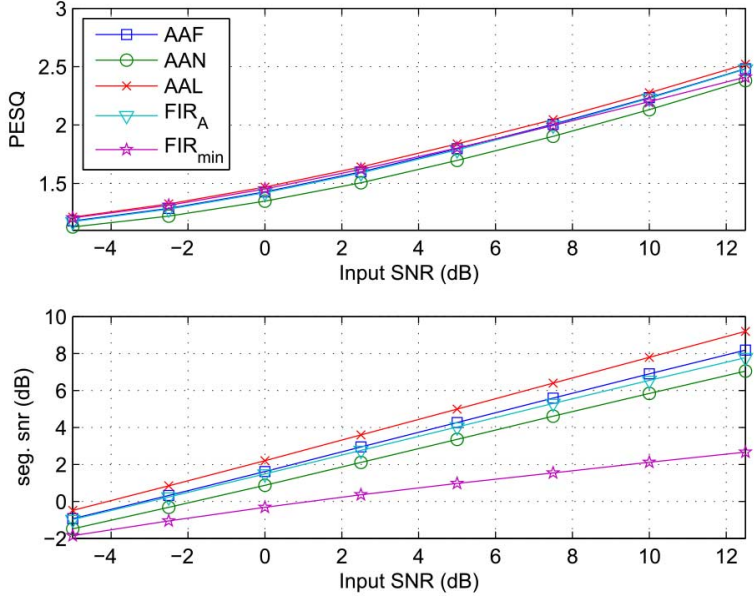

Fig. 11. Experimental results for different synthesis schemes for traffic noise.
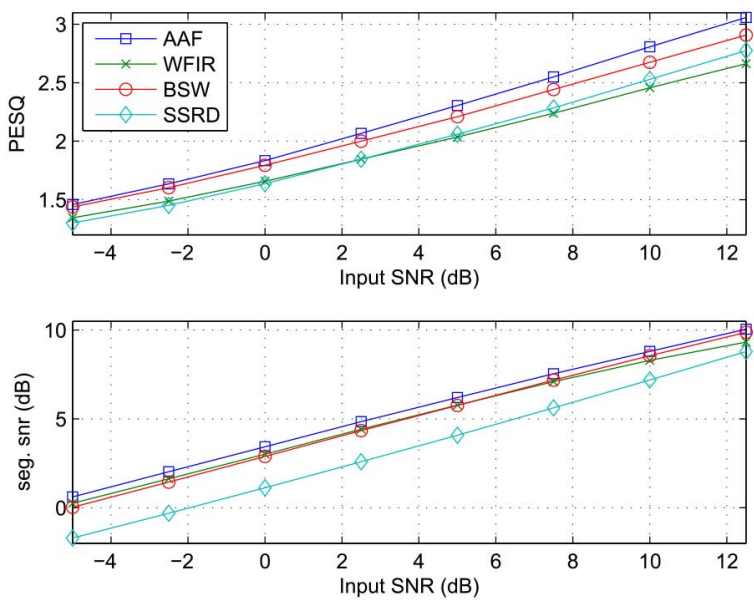

Fig. 12. Experimental results for different speech enhancement methods for car noise.

window in BSW is implemented using a high-order DFT analysis filter bank, which means the computational complexity is similar to AAF, while WFIR and SSRD have a lower computational complexity since they use a lower-order DFT. WFIR and BSW is implemented with the same Wiener gain with a-priori SNR estimation that AAF uses, while SSRD uses a spectral subtraction approach with spectrum-dependent adaptive averaging described in the paper [13]. Other related approaches, such as [14], [11], have not been considered in this comparison since they have a significantly higher delay and are therefore not suitable for low delay implementation. The methods have been tested on car noise, traffic noise and canteen noise and the results can be seen in Figure 12, Figure 13 and Figure 14 respectively. Among the test signals, it is seen that car noise has the
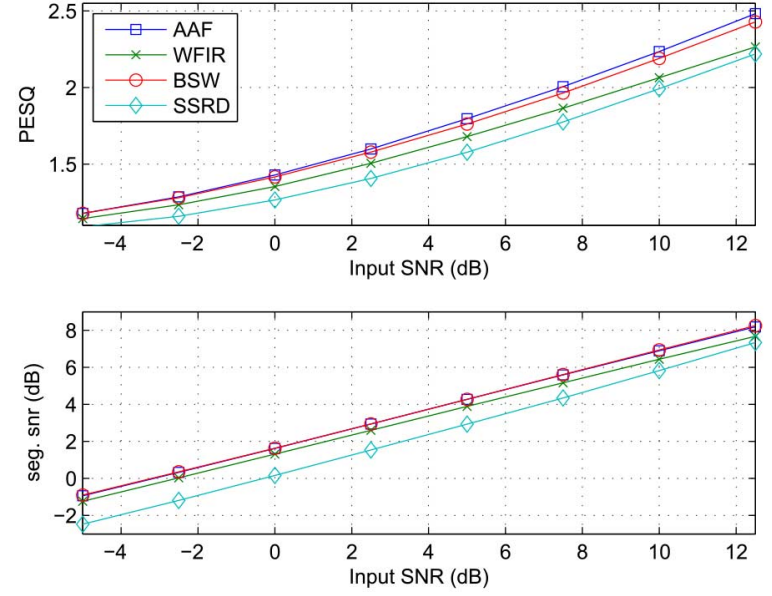

Fig. 13. Experimental results for different speech enhancement methods for traffic noise.
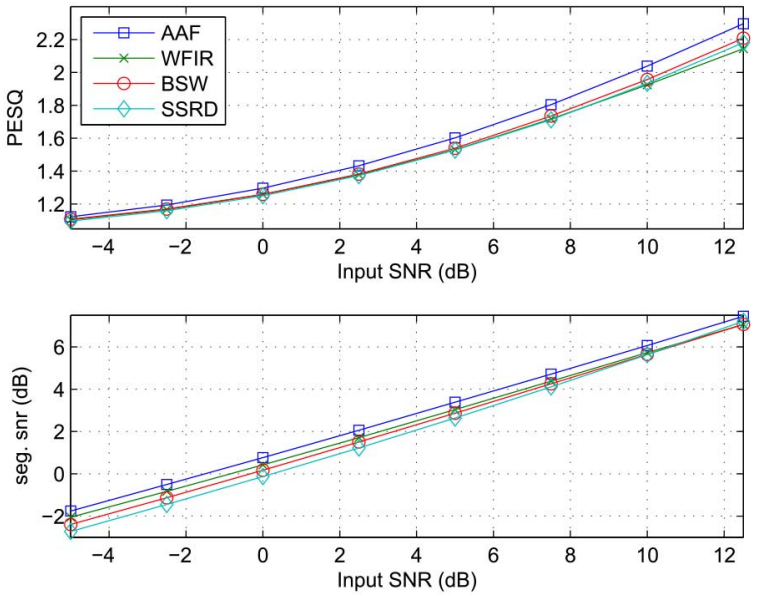

Fig. 14. Experimental results for different speech enhancement methods for canteen noise.

highest seg. SNR and PESQ scores and also has the greatest variation between the compared methods. In particular AAF and BSW performs better in terms of PESQ, while SSRD performs worse in terms of seg. SNR. The better PESQ scores of $\mathrm{AAF}$ and BSW can be explained by the higher frequency resolution in the analysis, while the lower seg. SNR score of SSRD is due to the reduced gain convolution that limits the ability to apply a steep cut in the frequency response. The PESQ results are in agreement with informal listening tests. The same trend is seen for traffic and canteen noise, although the scores are lower and have less variation. This is explained by the fact that traffic and especially the canteen noise signal is a harder problem due to the highly modulated content in the signal. A modulated noise signals reduces the advantage of having a high 
frequency resolution, since the signal is not stationary over the corresponding longer analysis window. Overall it is seen that the proposed method has the best performance, in particular in terms of objective perceptual performance as measured by PESQ.

\section{Conclusion AND Future Outlook}

An adaptive TF analysis/synthesis scheme for processing of audio with a low delay from 0 to $4 \mathrm{~ms}$ and low computational complexity has been proposed in this paper. The scheme is suitable for real-time sound devices such as mobile communication devices or hearing aids. The scheme can obtain a nonuniform frequency resolution with any desired bandwidth for each frequency band. It can adapt the TF distribution independently in each frequency band according to the signal to obtain a time-varying TF analysis. A number of adaptive TF analysis schemes have been described that are suitable for a real-time implementation. Synthesis of the gain-modified signal with a low delay is achieved using an asymmetric synthesis window that results in a mixed phase filter bank that maintains the low delay of a reference filter bank but with an improved frequency response. Further reduction in the delay is achieved by applying the gain to the signal as a time-varying FIR filter. A noise reduction experiment was performed to show the proposed scheme's good performance compared to reference methods with the same delay. The computational complexity was shown to be dominated by the FFT algorithm in the DFT filter bank and is therefore mainly determined by the number of frequency bands and the chosen FFT algorithm.

Future research includes investigating alternative ways to use the information from the adaptive TF analysis to further improve the processing of the audio signal. Many real-time audio processing applications, such as hearing loss compensation, frequency transposition and acoustic echo cancellation utilize a TF distribution and it should be investigated how such an adaptive analysis/synthesis scheme can improve their performance. Another interesting area of research is the estimation of the adaptive TF analysis and how the delay inherent in the analysis affects the application of the gain function.

\section{REFERENCES}

[1] J. Agnew and J. M. Thornton, "Just noticeable and objectionable group delays in digital hearing aids," J. Amer. Acad. Audiol., vol. 11, no. 6, pp. 330-336, 2000.

[2] J. M. Kates and K. H. Arehart, "Multichannel dynamic-range compression using digital frequency warping," EURASIP J. Appl. Signal Process., vol. 2005, pp. 3003-3014, Jan. 2005.

[3] M. Vetterli and J. Kovačevic, Wavelets and Subband Coding. Englewood Cliffs, NJ, USA: Prentice-Hall, 1995.

[4] R. Crochiere and L. Rabiner, Multirate Digital Signal Processing. Englewood Cliffs, NJ, USA: Prentice-Hall, 1983.

[5] P. P. Vaidyanathan, Multirate Systems and Filter Banks. Englewood Cliffs, NJ, USA: Prentice-Hall, 1993.

[6] J. M. Kates, Digital Hearing Aids. San Diego, CA, USA: Plural Publishing, 2008.

[7] H. W. Löllmann and P. Vary, "Low delay filter-banks for speech and audio processing," in Speech and Audio Processing in Adverse Environments, E. Hänsler and G. Schmidt, Eds. New York, NY, USA: Springer, 2008, pp. 13-61.

[8] L. Cohen, "Time-frequency distributions-a review," Proc. IEEE, vol. 77, no. 7, pp. 941-981, Jul. 1989.
[9] M. Liuni, A. Robel, E. Matusiak, M. Romito, and X. Rodet, "Automatic adaptation of the time-frequency resolution for sound analysis and resynthesis," IEEE Trans. Audio Speech Lang. Process., vol. 21, no. 5, pp. 959-970, May 2013.

[10] H. Sepehr, A. Y. Nooralahiyan, and P. V. Brennan, "Improving performance of a noise reduction algorithm by switching the analysis filter bank," in Image and Signal Processing, A. Elmoataz, O. Lezoray, F. Nouboud, D. Mammass, and J. Meunier, Eds. New York, NY, USA: Springer, 2010, vol. 6134, pp. 262-271.

[11] D. Rudoy, P. Basu, and P. Wolfe, "Superposition frames for adaptive timefrequency analysis and fast reconstruction," IEEE Trans. Signal Process., vol. 58, no. 5, pp. 2581-2596, May 2010.

[12] D. Mauler and R. Martin, "A low delay, variable resolution, perfect reconstruction spectral analysis-synthesis system for speech enhancement," in Proc. 15th Eur. Signal Process. Conf. (EUSIPCO'07), Poznan, Poland, 2007, pp. 222-226.

[13] H. Gustafsson, S. Nordholm, and I. Claesson, "Spectral subtraction using reduced delay convolution and adaptive averaging," IEEE Trans. Speech Audio Process., vol. 9, no. 8, pp. 799-807, Nov. 2001.

[14] R. Hendriks, R. Heusdens, and J. Jensen, "Adaptive time segmentation for improved speech enhancement," IEEE Trans. Audio Speech Lang. Process., vol. 14, no. 6, pp. 2064-2074, Nov. 2006.

[15] P. Loughlin, J. Pitton, and B. Hannaford, "Approximating time-frequency density functions via optimal combinations of spectrograms," IEEE Signal Process. Lett., vol. 1, no. 12, pp. 199-202, Dec. 1994.

[16] P. Loughlin, J. Pitton, and B. Hannaford, "Fast approximations to positive time-frequency distributions, with applications," in Proc. Int. Conf. Acoust. Speech Signal Process. (ICASSP'95), May 1995, vol. 2, pp. 1009-1012.

[17] D. Mauler and R. Martin, "Improved reproduction of stops in noise reduction systems with adaptive windows and nonstationarity detection," EURASIP J. Adv. Signal Process., vol. 2009, no. 1, p. 469-480, 2009.

[18] K. Andersen and M. Moonen, "An adaptive time-frequency analysis scheme for improved real-time speech enhancement," in Proc. IEEE Int. Conf. Acoust. Speech Signal Process. (ICASSP), May 2014, pp. 62656269.

[19] H. Sorensen, D. Jones, M. Heideman, and C. Burrus, "Real-valued fast fourier transform algorithms," IEEE Trans. Acoust. Speech Signal Process., vol. 35, no. 6, pp. 849-863, Jun. 1987.

[20] M. Vetterli and D. Le Gall, "Perfect reconstruction FIR filter banks: Some properties and factorizations," IEEE Trans. Acoust. Speech Signal Process., vol. 37, no. 7, pp. 1057-1071, Jul. 1989.

[21] X.-G. Xia and B. Suter, "FIR paraunitary filter banks given several analysis filters: Factorizations and constructions," IEEE Trans. Signal Process., vol. 44, no. 3, pp. 720-723, Mar. 1996.

[22] K. Eneman and M. Moonen, "DFT modulated filter bank design for oversampled subband systems," Signal Process., vol. 81, no. 9, pp. 19471973, 2001.

[23] H. Bolcskei and F. Hlawatsch, "Oversampled cosine modulated filter banks with perfect reconstruction," IEEE Trans. Circuits Syst. II: Analog Digit. Signal Process., vol. 45, no. 8, pp. 1057-1071, Aug. 1998.

[24] N. Grbic, J. de Haan, I. Claesson, and S. Nordholm, "Design of oversampled uniform DFT filter banks with reduced inband aliasing and delay constraints," in Proc. 6th Int. Symp. Signal Process. Appl., vol. 1, 2001, pp. 104-107.

[25] H. Dam, S. Nordholm, A. Cantoni, and J. de Haan, "Iterative method for the design of DFT filter bank," IEEE Trans. Circuits Syst. II: Exp. Briefs, vol. 51, no. 11, pp. 581-586, Nov. 2004.

[26] K. F. C. Yiu, N. Grbic, S. Nordholm, and K. L. Teo, "Multicriteria design of oversampled uniform DFT filter banks," IEEE Signal Process. Lett., vol. 11, no. 6, pp. 541-544, Jun. 2004.

[27] D. Hermann, E. Chau, R. Dony, and S. Areibi, "Window based prototype filter design for highly oversampled filter banks in audio applications," in Proc. IEEE Int. Conf. Acoust. Speech Signal Process. (ICASSP'07), Apr. 2007, vol. 2, pp. II-405-II-408.

[28] H. W. Löllmann and P. Vary, "Generalized filter-bank equalizer for noise reduction with reduced signal delay," in Proc. Eur. Conf. Speech Commun. Technol. (Interspeech), Lisbon, Portugal, Sep. 2005.

[29] H. W. Löllmann and P. Vary, "Low delay filter for adaptive noise reduction," in Proc. Int. Workshop Acoust. Echo Noise Control (IWAENC), Sep. 2004, pp. 205-208.

[30] O. Herrmann and W. Schuessler, "Design of nonrecursive digital filters with minimum phase," Electron. Lett., vol. 6, no. 11, pp. 329-330, May 1970.

[31] N. Damera-Venkata, B. Evans, and S. McCaslin, "Design of optimal minimum-phase digital FIR filters using discrete hilbert transforms," IEEE Trans. Signal Process., vol. 48, no. 5, pp. 1491-1495, May 2000. 
[32] Y. Ephraim and D. Malah, "Speech enhancement using a minimummean square error short-time spectral amplitude estimator," IEEE Trans. Acoust. Speech Signal Process., vol. 32, no. 6, pp. 1109-1121, Dec. 1984.

[33] T. Gerkmann and R. Hendriks, "Unbiased MMSE-based noise power estimation with low complexity and low tracking delay," IEEE Trans. Audio Speech Lang. Process., vol. 20, no. 4, pp. 1383-1393, May 2012.

[34] P. C. Loizou, Speech Enhancement: Theory and Practice. Boca Raton, FL, USA: CRC Press, 2013.

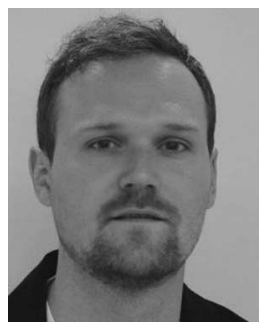

Kristian Timm Andersen (S'13) was born in Herlev, Denmark, in 1983. He received the M.Sc. degree in electrical engineering from the Technical University of Denmark, Lyngby, Denmark, in 2008 $\mathrm{He}$ is currently pursuing the Ph.D. degree in electrical engineering, KU Leuven, Leuven, Belgium. He is a member of KU Leuvens STADIUS Group. His research interests include audio signal processing, acoustical signal enhancement, and real-time audio applications. From 2008 to 2013, he was an R\&D DSP Engineer with Widex, A/S, Lynge, Denmark, where he developed novel and patentable DSP algorithms for hearings aids.

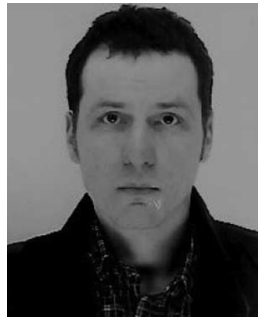

Marc Moonen (M'94-SM'06-F'07) is a Full Professor with the Department of Electrical Engineering, KU Leuven, Leuven, Belgium, where he is heading a research team working in the area of numerical algorithms and signal processing for digital communications, wireless communications, DSL, and audio signal processing. He was the Chairman of the IEEE Benelux Signal Processing Chapter (1998-2002), a member of the IEEE Signal Processing Society Technical Committee on Signal Processing for Communications, and the President of EURASIP (European Association for Signal Processing, from 2007-2008 and from 2011-2012). He has served as the Editor-in-Chief for the EURASIP Journal on Applied Signal Processing (2003-2005), an Area Editor for Feature Articles in IEEE Signal Processing Magazine (2012-2014), and has been a member of the Editorial Board of the IEEE TRANSACTIONS ON CIRCUITS AND SYSTEMS II, the IEEE Signal Processing Magazine, Integration-the VLSI Journal, and EURASIP Journal on Wireless Communications and Networking, and Signal Processing. He is currently a member of the Editorial Board of EURASIP Journal on Advances in Signal Processing. He was the recipient of the $1994 \mathrm{KU}$ Leuven Research Council Award, the 1997 Alcatel Bell (Belgium) Award (with Piet Vandaele), the 2004 Alcatel Bell (Belgium) Award (with Raphael Cendrillon), and was a 1997 Laureate of the Belgium Royal Academy of Science. He was also the recipient of the Journal Best Paper Awards from the IEEE Transactions on Signal Processing (with Geert Leus and with Daniele Giacobello) and from Elsevier Signal Processing (with Simon Doclo). 